\title{
Community Introduction of Practice Parameters for Autistic Spectrum Disorders: Advancing Early Recognition
}

\author{
Laurent Holzer, ${ }^{1,3}$ Raluca Mihailescu, ${ }^{2}$ Catherine Rodrigues-Degaeff, ${ }^{1}$ Laurent Junier, ${ }^{1}$ Carole \\ Muller-Nix, ${ }^{1}$ Oliver Halfon, ${ }^{1}$ and François Ansermet ${ }^{1}$
}

Objectives: Within a strong interdisciplinary framework, improvement in the quality of care for children with autistic spectrum disorders through a 2 year implementation program of Practice Parameters, aimed principally at improving early detection and intervention. Method: We developed Practice Parameters (PPs) for Pervasive Developmental Disorders and circulated the PPs to all child and adolescent psychiatrists practicing in the region. Results: PP development and parallel information strategies resulted in a significant decrease of 1.5 years in the mean-age-at-diagnosis. However, further analysis indicated that improvement was only transient. Conclusion: Despite the encouraging improvement in mean-age-atdiagnosis 2 years after PP implementation, other indicators showed a failure to maintain the improvements. A systematic screening program would be the most reliable method to reinforce the PPs.

KEY WORDS: Autism; Pervasive Developmental Disorder; early detection; community; quality of care.

\section{INTRODUCTION}

Autistic spectrum disorders, among the most challenging of child psychiatric problems, severely affect development and carry major risk of chronic impairment and disability. Such poor prognosis requires clinicians to be active in the early phase of child development, aiming for prompt detection and rapid application of research findings to clinical practice. While recent reports suggest an advance in understanding the physiopathology of ASDs,

\footnotetext{
${ }^{1}$ Hôpital Nestle, Service Universitaire de Psychiatrie de l'Enfant et de l'Adolescent (SUPEA), Lausanne, Switzerland.

${ }^{2}$ Département de Psychologie, Université de Genève, Genève, Switzerland.

${ }^{3}$ Correspondence should be addressed to: Laurent Holzer, Hôpital Nestle, Service Universitaire de Psychiatrie de l'Enfant et de l'Adolescent (SUPEA), CTJA/48 avenue de Beaumont, 1012, Lausanne, Switzerland; Tel: +41-21-314-1730; Fax: +41-21-3141735; e-mail: Laurent.Holzer@inst.hospvd.ch
}

treatment issues remain controversial and the incidence rate continues to rise (Fombonne, Simmons, Ford, Meltzer, \& Goodman, 2001; Gillberg \& Wing, 1999).

Despite the expanded efforts in western countries to advance early intervention, the mean-age-atdiagnosis remains too high. Even in areas that use systematic screening and follow-up, there is a real gap between the kind of careful screening done in research programs and the clinical reality of age at referral. Fombonne et al. mention that whereas parental concerns occur between 15 and 22 months of age, the first contact with a specialist takes place nearly a half-year later, between 20 and 27 months. Then it is not until a mean age of six years that these children receive a highly specialized assessment (De Giacomo \& Fombonne, 1998). A recent report also raised the issue of disparities between mean-ages-atdiagnosis for two racial groups: white children were diagnosed as autistic at a mean 6.3 years of age, 
compared with 7.9 years for African-American children (Mandell, Listerud, Levy, \& Pinto-Martin, 2002).

Growing research data, multiple etiology hypotheses, and a variety of clinical expressions of these disorders often leave the clinician overwhelmed by information, yet paradoxically lacking in relevant practice knowledge. While many studies on screening and early intervention lead one to believe that diagnosis at a younger age is achievable, what concrete methods can a clinician use to gain earlier access to these children and improve their prognoses? While translating scientific evidence into clinical practice remains a complex and challenging goal for authors of clinical guidelines (Davis \& Taylor-Vaisey, 1997), useful Practice Parameters (PPs) should fit a clinician's expectations as well as improve the quality of care of autistic children.

\section{CONTEXT}

\section{The Regional PDD Situation}

Autism and related pervasive developmental disorders have an estimated minimum prevalence of 18.7 per 10,000 (Fombonne, 1999). This should lead to an incidence of approximately 20 new cases a year for the Swiss canton of Vaud, which has approximately 8000 births per year. Approximately 30-40 new cases of ASD present to the Lausanne University Child and Adolescent Psychiatry Service (UCAPS) facilities each year. See Appendix B.
There is no systematic screening for ASDs in the canton. Between 9 months and 4 years of age, children are not required to see a physician (for vaccinations, for example). The Lausanne UCAPS includes 41 of the 103 child psychiatrists practicing in the canton and offers a specialized joint consultation for ASDs. It also maintains some 100 places for providing institutional care in a day hospital. Child psychiatrists do not necessarily see all the children who present with symptoms of ASD. Considering the nature of the problems that require assessment and specialized care, most cases of ASDs are referred to the public health care sector covered by UCAPS, while the private sector is overloaded and has long waiting lists of patients seeking appointments. Case numbers and mean-age-at-diagnosis over a 4 year period (1996-1999) were as follows: (see Table I).

In an area where nursery school can begin at the age of 4 and obligatory primary school starts at the age of 6 , the mean-age-at-diagnosis is slightly over 6 years. This is the age at which the child is first seen in the service, with the diagnosis following within approximately 1 month. Nearly all of these children are referred to the UCAPS by their pediatrician or their school (based on suspicion of ASD), prior to actual diagnosis.

Thus, the principal problem identified was a mean-age-at-diagnosis that was particularly high in comparison with other studies (Baron-Cohen, Allen, \& Gillberg, 1992; Gray \& Tonge, 2001). Most authors believe that early detection and treatment are important to ensure the best treatment results. It is generally accepted that early acquisition of communicative and

Table I. New Cases of PDD Over a 4 Year Period (1996-1999)

\begin{tabular}{lcc}
\hline Diagnosis* & $n$ & Mean age (in years) \\
\hline Childhood autism & 15 & 4.9 \\
Atypical autism & 17 & 5.2 \\
Rett's syndrome & 0 & - \\
Other childhood disintegrative disorder & 10 & 7.0 \\
Hyperactive disorder associated with mental retardation and stereotyped movements & 0 & 7 \\
Asperger's syndrome & 46 & 7.7 \\
Other pervasive developmental disorders & 81 & 6.1 \\
Pervasive developmental disorder not otherwise specified & Total $n=176 \quad$ Grand $M=6.1$ years \\
\end{tabular}

*Patients are diagnosed by senior psychiatrists according to the "ICD 10 criteria" (clinical descriptions and diagnostic guidelines). Use of valid diagnostic tools is not systematic.

The unusual diagnostic distribution with an overrepresentation of PDD-NOS and "other PDD" is certainly a consequence of transcoding. CFTMEA [the French classification of mental disorders of children and adolescents-(Misès et al., 1988)] recommends the PDD-NOS transcoding for what they call "psychotic disharmony," a quite frequent and popular diagnosis in French-speaking, psychoanalytically oriented child psychiatry. Experienced clinicians familiar with the CFTMEA tend to refer to the transcoding recommendation instead of the ICD 10 criteria themselves. 
representational skills influences later verbal and social capacities. This emphasizes the importance of early intervention to improve the development of these fundamental precursors (Robins, Fein, Barton, \& Green, 2001; Rutter, 1999; Sigman, 1999). From this point of view, mean-age-at-diagnosis is an important indicator, and lowering this age becomes a prerequisite for improvement in the quality of care.

\section{Disparities in Clinical Practice}

Clinical practice cases show that the care process can be disrupted at several stages. At the detection phase, there is a tendency to minimize or to diagnose ASDs only as a last resort. We observed wide variations in clinical practice at the diagnostic assessment phase (which is often long and sometimes insufficiently coordinated) and the treatment planning level (using criteria that do not necessarily take into account either medical indications or scientific evidence). Certain children benefited from a thorough neuro-pediatric and/or child psychiatric assessment. Others were integrated into care structures or schools without any specialized medical assessment.

\section{A Joint Consultation of Neuro-Pediatrics and Child Psychiatry (JCNC)}

As autism and PDD are neuro-psychiatric developmental disorders, both child neurologists and child psychiatrists are involved in this field. In clinical dayto-day practice, parents and children deal with one medical specialist rather than with two or more professionals. We attempted to remedy this common division of care management through a joint consultation for children suffering from developmental disorders. This integrates the biological, neuro-psychological, and child psychiatric points of view into a multidisciplinary perspective, and provides a treatment plan that takes into account these different aspects. A child neurologist, a child psychiatrist, and a psychologist conduct this joint consultation.

\section{The Regional Health Service's "Quality Program"}

Promoting the quality of care is part of the mission of our regional health service, which launched a Quality Program across the various care providers (Graz, Vader, Burnand, \& Paccaud, 1996). Quality management in health care aims to improve health outcomes, but is still under development in the current Swiss context. Economic issues and restrictions are also part of quality management in health care projects, although not to the extent that we observe in the USA context (Jellinek, 1999).

\section{OBJECTIVES}

We decided to develop and implement PPs for early diagnosis and treatment of children with ASDs. Our aim was to improve the clinical system in the region by reducing mean-age-at-diagnosis so as to improve long term outcomes, increase referrals to child psychiatrists, and improve the clinical identification process. The goal of our PPs was to provide clear recommendations, give an overall view of the questions, and standardize practices of detection, diagnosis, and treatment planning for children up to 4 years old who present developmental problems. We placed special emphasis on developing concrete measures, as PPs are operationally grounded in the clinical field. Given the potentially negative label of autism/Pervasive Developmental Disorders (ASDs), we focused on early detection of children at risk for $A S D s$ rather than children with ASDs.

Generally, our pilot program objectives were not strictly part of a clinical research project aimed at evaluating an original hypothesis on autism and PDD. Rather, we sought to test the following hypothesis: the development and implementation of PPs allows for improved quality of care in the ASD clinical field by improving certain indicators, notably the mean-age-at-diagnosis.

\section{METHOD}

Our quality care assurance project was a twoyear (April 1998-April 2000) action research program to develop and implement PPs supported by the promotion of active interdisciplinary coordination for early detection and diagnosis of ASDs. In order to encourage and develop a culture of "quality orientation" within the academic hospital services, the project directors worked to improve the quality of care by providing financial support. Complicated care pathways and complex clinical problems posed by children presenting ASDs focussed the project on developing PPs. Weekly meetings with JCNC clinicians anchored this project in the concrete reality the group's goal to become a cantonal reference for ASD issues. The complexity of care networks and the personal and institutional stakes of the professionals involved in ASDs engendered a variety of reactions 
that required many adjustments in setting up the project. In spite of these obstacles, a pragmatic approach gradually developed to tighten the objectives of improving early identification and follow-up.

The evaluation of the impact of PPs circulation was conducted by analyzing the medical files of the JCNC and the other divisions of the service, as well as by analyzing statistical data gathered for all the children followed in the service. The second author (RM, independent from the UCAPS) consulted the files of all 69 children for whom the service had made a ASD diagnosis in 1999 and 2000. She examined the trajectory of care, the clinical assessment performed, and the treatment plan proposal when available. We systematically summarized other data, such as investigation requests and telephone advice based on clinical descriptions.

Mean age at diagnosis was our main indicator. Data came from computerized medico-administrative files, which staff members complete within the month following admission of the child. We hypothesized that the intervention program would also increase the referral of children with a suspicion of ASD to the JCNC or to a child psychiatry consultation, and that children with ASDs would be more likely to receive a complete assessment.

Other indicators we used were operationalized as follows:

1. The number of children benefiting from a complete assessment. We considered a case to be complete when the child had benefited from a child psychiatrist consultation, a pediatric consultation, a formal audiologic assessment, a psychometric evaluation, and at least one standardized and validated diagnostic tool.

2. Use of the PPs by the clinicians: We considered the PPs to have been followed when the case was complete and all five stages mentioned in point \#II) had been achieved and at least one of the recommended tools in points \#2 and \#3 had been used.

3. Number of children referred by practicing pediatricians: information contained in the medical file.

4. Number of children referred to the JCNC: activity of the JCNC that appears in the medical statistics of the UCAPS.

5. Number of children referred to the JCNC based on a suspicion of PDD.

6. Number of PDD diagnoses confirmed.

\section{Development of PPs}

We developed our PPs by scrupulously following the twenty-two PP guidelines developed by Burnand (1999) for the Center of Clinical Epidemiology. We compiled information from two research databases (MEDLINE and PSYCLIT), from Internet sites dedicated to ASDs, and from numerous ASD articles, books, and other works. PPs must consider the local context in which they are developed (theoretical orientation, practitioners' training, local resources in specific specialties, educational institutions, existing therapeutic practices, etc.). We submitted the PPs for evaluation to three external experts: Prof. Isabelle Rapin, Prof. Bernard Golse, and Prof. Pierre Ferrari. Concrete clinical results in a community-based setting were the underlying incentive for our approach, rather than the simple development of PPs. In seeking to lower the mean-age-at-diagnosis, we adopted an information strategy aimed at professionals involved with children at risk for ASDs.

\section{Publication and Circulation of the PPs}

The PPs were issued throughout the region along with an explanation of the goals of our approach. The PPs addressed early detection and diagnosis, diagnostic investigations, and treatment plans. See Appendix A.

\section{Early Detection and Diagnosis}

ASDs had not been a target for systematic regional screening. Moreover, the Swiss health care system does not facilitate early screening strategies, as pediatrician visits are not systematically conducted between the ages of 9 months and 4 years. On the other hand, early signs of autism and other PDDs are neither pathognomonic nor even specific [i.e., sleep disturbances, gaze monitoring abnormalities, low interest in communication (Tanguay, Robertson, \& Derrick, 1998)]. As non-specific signs can be found in many normal situations, they do not automatically warrant a specialized consultation; cost, availability of specialists, or even the anxiety generated in the parents and/or child are all dissuasive factors. However, given the potentially serious nature of these disorders, early intervention is warranted. Although the validity of early diagnosis and the efficacy of early treatment have not been formally demonstrated scientifically, there are converging arguments supporting the importance of early detection and treatment (American Academy of Child and Adolescent 
Psychiatry Official Action, 1999; Filipek et al., 1999, 2000; McEachin, Smith, \& Lovaas, 1993; Rutter, 1999; Sauvage \& Hameury, 1990; Sigman, 1999).

Our detection strategy involved the following sequential steps leading either to the JCNC or to another child psychiatry consultation.

1. Sensitization of the field, that is, familiarizing various early childhood professionals (nursery school personnel, day care workers, specialized social workers, educators) with developmental issues and with non-specific signs of possible ASDs problems, such as social withdrawal, abnormalities in social reciprocity, sharing enjoyment, directing attention, etc. To avoid a drift towards alarmist positions and abusive diagnosis, we emphasized that the identification by field professionals of non-specific signs should be merely a starting point for a consultation by a pediatrician or family doctor.

2. Informing pediatricians and general practitioners of a specific tool: the CHAT [Checklist for Autism in Toddlers, by Baron-Cohen et al. (1996)]. Our choice of this tool was motivated by its simplicity of use, precise item definitions, high specificity, and overall validity. Its sensitivity, however, was lower than expected, according to recent follow-up data to the age of seven (Baird et al., 2000). While it is not a diagnostic tool, it does identify children at risk. A risk profile of a child who presents non-specific signs should lead the pediatrician or primary care physician to refer the child to a child psychiatric consultation or to the JCNC.

3. Investigative steps (by the $\mathrm{JCNC}$ or a child psychiatrist) to confirm or invalidate the diagnostic hypothesis of a child at ASD risk, and to prepare a treatment plan for confirmed cases.

\section{Diagnostic Investigations}

A number of steps were formalized in accordance with the recommendations developed by the AACAP (American Academy of Child and Adolescent Psychiatry Official Action, 1999) and are described in Appendix A.

We emphasized the importance of a detailed assessment that evaluated a variety of factors, such as medical history, pediatric examination, child psychiatric observation, neurological assessment, psychological assessment, laboratory investigations, and the child's immediate domestic and socioeconomic environment. We recommended the use of standardized assessment tools, such as the Childhood Autism Rating Scale (Schopler, Reichler, DeVellis, \& Daly,
1980), the Pre-Linguistic Autism Diagnostic Observation Schedule (Dilavore, Lord, \& Rutter, 1995) and the Psycho-Educational Profile-Revised (Steernemann, Muris, Merckelbach, \& Willems, 1997). Despite limitations of the CARS regarding its unitary conception (Stella, Mundy, \& Tuchman, 1999) and a tendency to overdiagnose autism with the current cutoff score at 30 (Lord, 1997), this tool provide useful and reliable information at a broad community level. We also focused on symptom variation, notably as a function of age.

The psychiatrists' clinical impressions are a part of the assessment, and the final diagnosis will be based as much on the clinician's opinion as on the results of the assessment scales. Differentiating children at risk for autistic spectrum disorders from children with autistic spectrum disorders is a complex issue. From our point of view, no clear border can be established by any standardized tool. A clinical diagnosis of autism, confirmed with a diagnostic tool (CARS $>37$ for example), will lead to an autism diagnosis. A clinical impression of autism, not confirmed with any diagnostic tool (or a significant score on a diagnostic tool without clinical impression), is likely to moderate diagnosis certainty. In such cases, a diagnosis of atypical autism (CARS between 30 and 37) or PDD-NOS (CARS $<30)$ is given, and the "at risk" condition is reserved for very young children who do not meet all diagnostic criteria for an autistic spectrum disorder. We are dealing here with uncertainty coming from clinical age-related features with prognosis implications that are taken into account by the "at risk" notion. The establishment of the final diagnosis is a synthesis resulting from meetings of senior child psychiatrists, physicians who examined the child, and the psychologist who had tested the child.

\section{Treatment Plans}

At the end of the diagnostic assessment, a treatment plan was developed. The plan chosen depended largely on the wishes of the parents, and its orientation depended on the availability of alternatives in each therapeutic area.

No single treatment has been shown clearly superior to others in terms of therapeutic results (Gresham \& MacMillan, 1997). Nor are there formal elements that would permit us to exclude any particular form of treatment, arguing that it is ineffective or even harmful. Nor is there a clear consensus in favor of a specific method, but there is 
evidence to support a multidisciplinary therapeutic approach (Danion-Grilliat, 1996; Gilman \& Tuchman, 1995; Ozonoff \& Cathcart, 1998; Reid, Balis, \& Sutton, 1997; Rutter, 1999) and the effectiveness of educational interventions (Koegel, Koegel, \& McNerney, 2001; Ozonoff \& Cathcart, 1998). Further research is needed to evaluate the various therapeutic possibilities and their respective indications.

\section{Actions Undertaken}

We conducted a variety of activities aimed at advancing the early detection and diagnosis of ASDs. We made announcements and initiated discussions in various child care facilities, published articles in a targeted journal (Holzer, 2000; Rodrigues-Dagaeff et al., 1999), and circulated the Checklist for Autism in Toddlers (CHAT) (Baron-Cohen et al., 1996) to all 97 pediatricians of the Canton of Vaud and to 59 targeted general practitioners. We issued the PPs to all 103 child psychiatrists in the canton. The PPs were not issued to pediatricians and general practitioners because the volume of information was too great and the PPs dealt with a relatively specialized question for which most practitioners have "limited time." On the other hand, neuro-pediatricians and pediatricians in the development unit, who were direct partners in the Common Consultation (CCNP) project, did receive the PPs. An important project objective was to simplify the care pathways and to encourage specialized assessments by the CCNP or by a consulting child psychiatrist whenever possible.

At the end of the project, information about the PPs had been relayed and actively sustained through March 2000. PPs were systematically distributed every 6 months to all new physicians and psychologists in the UCAPS. The final report of the project with the PPs was available on the website of the Hospices Cantonaux (http://www.hospvd.ch/qualite/). However, the referral network had not been directly sustained as it had been during the project.

\section{RESULTS}

\section{Lowering Mean-Age-at-Diagnosis}

This is an indispensable first step for improving the level of care and establishing conditions for optimal treatment. It is the clearest indicator of the effectiveness of early detection and diagnosis efforts. We were thus encouraged that, following the introduction of the PPs, the mean-age-at-diagnosis dropped 1.5 years from age 6 to $4.5[p<.01]$ in 2000 (See Table II).

During the same period, the University of Geneva's child and adolescent psychiatry service (serving a population of about 414,000 with 4600 annual births) diagnosed 56 cases of autism or other PDDs. These had a mean age of 5.1 years that increased slightly between 1999 and 2000. Although these figures are not truly comparable, these data support the claim that the intervention program was responsible for the drop in mean-age at diagnosis in Vaud.

However, improvement was only transient; it disappeared as soon as the Quality Assurance in Health Care program ended.

\section{Other Indicators}

Variations in these indicators are shown in Table III. Slight improvements were indicated in the following areas: (a) increase in the number of children referred to consultation by external pediatricians, (b) increased consultation activity, notably for children suspected to have ASDs, (c) increase in the number of children benefiting from a complete assessment, and (d) application of the PPs by many practitioners.

Like mean-age-at-diagnosis, most of these indicators showed a return to previous levels after the program ended. The observed increase in referral to the JCNC stabilized while requests for expert opinion and telephone counseling increased.

\section{DISCUSSION}

\section{Interest in the Project}

Increasing interest in evidence-based medicine has lead to many developments of practice parameters (PPs). Despite their evolving scientific rigor and applicability, PP implementation in practice remains uncertain and their effects on quality of care are unclear and difficult to assess (Hayward, 1997). However, our Quality Assurance in Health Care program was instructive regarding its influence on day-to-day practice. This sort of intervention research attempts to translate research findings into practice and aims to improve the quality of health care in rough but significant indicators. Our type of program is likely to modify temporarily some clinical practices and shows some improvement in key quality of care indicators. It is also cost effective in that it uses existing resources (clinicians, service logistics, 
Table II. Mean-Age-at-Diagnosis of New Cases of PDD

\begin{tabular}{lccccc}
\hline & 1997 & 1998 & 1999 & 2000 & 2001 \\
\hline N of new PDD cases & 33 & 42 & 37 & 32 & 30 \\
Mean Age & $5.9( \pm 2.2)$ & $6.1( \pm 1.2)$ & $6.1( \pm 2.1)$ & $4.6^{*}( \pm 1.5)$ & $6.6( \pm 1.3)$ \\
\hline
\end{tabular}

$* p<.01$.

Table III. Variations of the Other Indicators

\begin{tabular}{lccccccrr}
\hline & $\begin{array}{c}\text { SUPEA N } \\
\text { of PDD }\end{array}$ & $\begin{array}{c}\text { Referred by } \\
\text { pediatricians }\end{array}$ & $\begin{array}{c}\text { JCNC } \\
\text { new cases }\end{array}$ & $\begin{array}{c}\text { JCNC N } \\
\text { of PDD suspicion }\end{array}$ & $\begin{array}{c}\text { JCNC N } \\
\text { of PDD }\end{array}$ & $\begin{array}{c}\text { Other } \\
\text { divisions }\end{array}$ & $\begin{array}{c}\text { Complete } \\
\text { assessment }\end{array}$ & $\begin{array}{c}\text { PP } \\
\text { followed }\end{array}$ \\
\hline 1999 & 37 & 14 & 83 & 47 & 19 & 18 & 9 & 4 \\
2000 & 32 & 18 & 101 & 53 & 22 & 10 & $19^{*}$ & $13^{*}$ \\
\hline$* p<.05$. & & & & & &
\end{tabular}

and information technology) coordinated by a psychologist whose position was the essential element financed by the project.

The PPs were an obvious support for clinicians, promoting early detection of ASDs and sensitizing the project partners to what was at stake. The implementation of PPs is an interesting and stimulating exercise, potentially capable of modifying daily attitudes and behaviors in the clinic, but in practice, it comes up against several obstacles.

As this project has primarily served the JCNC, it can be seen as an attempt to promote a child psychiatric approach in the field of ASDs. The regional context of Vaud, with a high density of child psychiatrists, makes the JCNC an operational solution. Delays for referral are much longer in child neurology and developmental pediatrics (a minimum of 6-9 months). Therefore, our aim to increase referral to child psychiatrists is certainly not to the detriment of child neurologist, developmental pediatrician, or psychologist. Child psychiatrists, given their high number in the canton, are an optimal resource for ASDs (looking beyond the common quarrels between medical specialties). As in other kinds of medical activity, the concept of "critical mass" must also be taken into account. Experience and expertise can only be acquired by seeing a sufficient number of children who present these kinds of problems. We place expertise on the side of medical art and not solely on the side of fundamental science, while recognizing that the two approaches are not exclusive. Indeed, each complements and enriches the other. The expertise behind this approach is one of an experienced clinic that integrates the most recent data in the ASD literature without assuming absolute certainty in ruling out all rare causes that could have a link to ASDs. The JCNC seemed ideally situated for progressively acquiring expertise and legitimated the effective support brought by the project.

\section{Limitations of the Methodology}

Several methodological limitations could reduce the general implications of our project. While the program was designed to serve the entire canton, it aimed above all to improve the identification and diagnosis of developmental problems in children. The methodology did not count all the children in the canton, nor did it measure the effect of the PP program on the children presenting ASD problems. Epidemiological data indicates that the number of children arriving at the UCAPS service is higher than the expected annual number of new cases. This suggests that UCAPS consultants eventually see most children with developmental disorders. While we strongly encouraged the use of better case identification strategies, we did not directly check to make sure they were followed, nor did we verify with pediatricians the manner in which they used the CHAT. It was through reviewing the patient files of UCAPS child psychiatrists that we verified the degree to which they followed recommendations (PPs).

We carried out the entire undertaking in a spirit of persuasion so as to promote increased surveillance and encourage early identification of suspected developmental disorders. A systematic assessment program would have required far greater resources, as well as an obligatory evaluative framework, in order to be effective. Moreover, a systematic screening and identification program would have been particularly difficult to set up, given the established patterns of 
health care practice and the organization of primary schooling in the canton.

Diagnostic reliability does not depend on a specific methodology systematically using a standardized diagnostic procedure or a validated diagnostic tool. Rather, it emerges from the work of senior psychiatrists who may or may not use specific diagnostic tools. This practice characteristic does not respond well to a requirement for standardized diagnostic procedures; it simply reflects the daily fact of clinical work in the "real world" of the Canton of Vaud.

The suspected tendency to diagnose autism or PDDs only when fairly certain, and confirmed by the child's development over several months or years, could explain much of the delayed diagnosis that we observe in our service. However, because of the requirement to diagnose within a month of admission, and knowing that there are no "waiting to see" diagnostic categories in the ICD 10, this hypothesis is not terribly plausible. The most commonly used PDD categories-PDD-NOS and "other PDD"-are general categories that can be prudently used to describe provisional suspicions of autism, contingent on a more refined diagnosis. While neither rigorous nor scientific, this attitude reflects how weaknesses in nosology may be used for clinical convenience.

\section{Diagnostic Accuracy}

The question of the reliability of early diagnosis has been raised by many authors (Filipek et al., 1999; Gray \& Tonge, 2001; Stone et al., 1999). The age at which it is possible to diagnose reliably autism or PDDs remains a controversial question. Several studies on this issue have postulated relative diagnostic stability at the age of 18 months (Baird et al., 2000). Importantly, not all DSM or ICD criteria can be fulfilled, because approximately 3 years of age are required for a complete picture of typical autism (Robins et al., 2001). While looking for reliable early diagnostic criteria that offer high predictive validity with good sensitivity and specificity, the notion of autistic risk or risk of ASDs seems to us an interesting compromise. It does not prematurely shut the child into a state of ASD, but allows for a more open prognosis while underscoring the importance of early intervention aimed at reducing the risk of evolution toward ASDs. However, this compromise is not systematic; it remains optional according to the clinician's own perception.
Promising new tools for early identification suggest that systematic screening and follow-up will develop and become much more reliable. Whereas initially the large-scale use of the CHAT spurred great interest and high hopes, the long-term followup revealed weaker sensitivity than foreseen for this diagnostic tool. In an attempt to remedy this problem, a "Modified CHAT" version was recently developed (Robins et al., 2001). The initial results appear to be encouraging; they confirm that systematic diagnosis is both feasible and increasingly reliable. The legitimate fears about false positives (incorrect early diagnosis of ASD) should be mitigated by the fact that the children tested present a range of developmental problems for which some kind of care is justified. The risk of diagnosing as pathological a developmental pattern that is simply a variant of the normal appears weak to us. In almost all cases, it is more the specific diagnosis of autism or some other developmental disorder that challenges clinicians, rather than a broader diagnosis of developmental difficulty. It is, of course, important that the reliability of specific diagnoses in autism spectrum disorders improves to offer equally specific care and treatment options. It would be unrealistic to advance systematic screening and diagnosis without the support of PPs, because diagnosis is only one of the (certainly fundamental) links in the total process of offering optimal care downstream from the diagnosis.

\section{Barriers to the Use of PPs}

Our results support the findings of Davis and Tailor-Vaisey, who have shown that the use of methods that are practice- and community-based rather than didactic are more effective in the adoption of clinical practice recommendations (Davis \& Taylor-Vaisey, 1997).

Barriers to the successful implementation of PPs and the achievement of beneficial secondary effects on health outcomes have been well-described (Hayward, 1997). Many difficulties have been reported concerning the application of PPs (Cabana et al., 1999). The following have been confirmed by clinician interviews: (a) unfamiliarity with the procedure, (b) the high volume of information, (c) the time required in order to understand and implement the program, (e) disagreements over "cookbook" guidelines, (f) the extra work involved, (g) low expectations concerning results, (h) a lack of motivation, and (i) the inertia of routine and habit. 
More specifically, the low level of proof in the ASD field seems limiting. An examination of the question leads to PPs being locally adapted, but a broader reach does not have strong grounding in evidence. Our PPs were intentionally indicative rather than directive. In order to advance flexibility in the use of PPs, we avoided guidelines that were too precise. As a result, the PPs themselves may be effective through a general sensitization rather than a specific application. Although we do not have empirical data to support this claim, a general approach may be less effective than a more specific and directive approach with a decision algorithm, such as the American Academy of Neurology has developed under the leadership of Filipek et al. (2000). A substantial limitation is the practical difficulty of treatment planning in the face of quotas in the receiving structures for children presenting ASDs. This can bring about disillusionment and lower expectations of concrete results. Many could easily question the efficacy of early detection and exhaustive assessment if there is no course of treatment for several years. However, the establishment of this common consultation was accompanied by a monitoring and medium-term ambulatory care structure for $0-3$ years. We considered that care downstream from the JCNC was assured, as this downstream care structure had never been saturated (in contrast to the long-term care structures serving school-aged children). The existence of this structure could have had a facilitating effect on the process of early diagnosis.

PP detection strategies based on key workers such as childhood professionals and pediatricians could implicitly lead to a passive attitude from child psychiatrists, whose roles are limited to the diagnostic and assessment phase. In other words, child psychiatrists have little to do with the detection phase; they simply wait for referrals by pediatricians and primary care physicians. A passive attitude replaces active collaboration through informal exchanges, networking, and reciprocal information, reinforces insularity between professions, and creates barriers to efficient care.

\section{Maintenance Strategies}

Although our pilot program might have perpetuated itself and become self-sufficient, our analysis suggests an evident failure of any self-maintenance effects. Maintenance strategies are crucial and require adequate resources. Cost offsets emerging from secondary prevention through early detection and treatment could supply resources for maintenance strategies. Apart from such marketing techniques such as promotion, reminders, and other communications, structural modifications in the child and adolescent mental health system could focus on early detection and diagnosis. Creating special appointments could reinforce links between child psychiatrists, pediatricians, and childhood professionals. Special research programs could translate fundamental research findings into clinical practice as suggested by the NIMH Blueprint for Change (Hoagwood \& Olin, 2002). Systematic screening strategies may also be considered and could be combined with the dissemination of PPs, which are an indispensable complement to systematic screening and diagnosis.

Despite the fact that Switzerland is wealthy and has a high rate of child and adolescent psychiatrists, children with ASDs are not necessarily well served. Differences between research settings and day-to-day practice lead to much inefficiency. The usual care processes need to be changed in depth, but child psychiatrists remain ineffective on their own. Sensitization and mobilization of child psychiatrists does not alone produce significant results in early detection. Primary care workers, early childhood workers, and pediatricians need to be closely involved. They are the best targets for early intervention programs to the extent that child psychiatrists are reliable downstream resources. Lack of outcome expectations has been identified as a barrier to adherence to clinical practice guidelines (Cabana et al., 1999) and is relevant for understanding resistance to change and pediatricians' skepticism toward child psychiatry. The many competing child psychiatric theories of autism, combined with limited care options and modest treatment results, certainly reinforce pediatric doubt. Further research is needed on the effectiveness of evidence-based treatment, translated into day-today practice, in order to increase child mental health outcome expectations and the confidence of our partners.

Changing clinical behavior is a complex and difficult task. Many strategies have been proposed, ranging from inducement to coercion. There is little doubt that the pharmaceutical industry applies some of the most effective inducement techniques aimed at modifying clinical behavior. Given the high cost and the relatively aggressive nature of such techniques, they cannot easily be transferred into a sensitive field such as childhood mental health, but they may be considered. Coercive techniques come more from insurance companies and managed care groups that address economic issues, which are often in conflict 
with improving individual clinical care (Jellinek \& Little, 1998).

\section{CONCLUSION}

Are PPs a useful solution for delayed diagnosis? Identified practice disparities lead to remediation attempts and PPs appear to be a potentially effective symptomatic treatment. PP development in the complex field of ASDs (where evidence-based recommendations suffer from a lack of reliable hard data) has engendered cautiousness and modesty. This limitation elicits open recommendation of PPs similar to those developed by the AACAP. Including specificity of the local context while keeping a broad and general usefulness requires compromise and a flexible theoretical orientation. This will produce a greater likelihood of application and use. On the other hand, too general a consideration and too little theoretical background could be a barrier to adherence by lowering expectations of health outcomes. As part of a broad sequential and interdisciplinary chain, early intervention is only the first link in the chain. It is necessary but not sufficient.

Although several methodological limitations are seen in this program, its strengths derive from outcome assessments in day-to-day practice with a simple but informative indicator such as mean-ageat-diagnosis. Although difficult to conduct and in some ways disappointing, this program has highlighted inadequacies and has confirmed chronic trends. Potential practice improvements, although temporary in our program, critically underline the need for broader implementation strategies and maintenance of PPs through active interdisciplinary collaboration and politically supported actions. A persuasive approach such as we have developed in this pilot program seems feasible and reasonably effective. For greatest effectiveness, it should be associated as much as possible with a systematic ASD screening and maintenance strategy.

\section{AKNOWLEDGMENTS}

This work was supported by a Quality in Health Care Program grant. The authors would like to thank Prof. B. Golse, Prof. Ferrari, and Prof. I. Rapin for their contribution to the Practice Parameters revision. Our special thanks go to Dr. B. Burnand, who provided us with guidelines for establishing the PPs.

\section{APPENDIX A}

The PPs were circulated along with all accompanying steps and the development methodology. For greater clarity, we limited ourselves to the presentation of the clinical portion of the document.

\section{Early Detection and Diagnosis}

Our detection strategy involved the following sequential steps leading either to a child psychiatry consultation or to the JCNC.

1. Sensitization of the field, that is, familiarizing various early childhood professionals (nursery school personnel, day care workers, specialized social workers, educators) with developmental disorders and with non-specific signs of possible ASDs, such as social withdrawal, abnormalities in social reciprocity, sharing enjoyment, directing attention etc. We decided against using a specific tool in order to reduce the risk of drifting towards alarmist positions and abusive diagnosis. This was also important in that the childhood facilities had no direct supervision from the initiators of the PPs. The initial choice for circulating an easily useable tool was reviewed, and direct intervention by professionals was favored (conference debates on this theme, presentations on the course of elaboration of the PPs). We emphasized that the identification by field professionals of nonspecific signs should be merely a starting point for a consultation by a pediatrician or family doctor. This course of sensitization constituted the first step.

2. Informing pediatricians and general practitioners of a specific tool: the CHAT (Checklist for Autism in Toddlers). Our choice of this tool was motivated by its simplicity of use, precise item definitions, high specificity, and overall validity. Its sensitivity, however, was lower than expected, according to recent follow-up data to the age of seven. While it is not a diagnostic tool, it does identify children at risk. A failed CHAT (as it reveals a risk profile for a child who presents non-specific signs) should lead the pediatrician or primary care physician to refer the child either to a child psychiatry consultation or to the JCNC. This kind of screening constituted the second step.

\section{Diagnostic Investigation}

Various steps were formalized and were generally in accordance with the recommendations developed by the AACAP. All diagnostic and assessment 
instruments mentioned were used by the JCNC and remain indicative.

\section{Pediatric Examination}

The first step of the consultation involved taking a medical and psychological history of the child and a description of the main presentation problems. The ADI-R (Autism Diagnostic Interview Revised) could be used at that point or later.

Historical Data were Notably Concerned with:

(a) the pregnancy, the perinatal period and the developmental history that takes into account the pregnancy, the delivery and neonatal period. Special attention was paid to the development stages of communication, language, motor milestones, as well as to the environmental responses. The question to the parents about their first recognition of troubles was very important: When did they first start to worry about the development of their child and why?

(b) The medical history: a ruling out of epilepsy, sensorial deficits, or medical signs evoking a specific disorder ( $\mathrm{X}$ fragile for example). The question of previous pharmacological treatment was important.

(c) The family history was reviewed for the presence of other autism spectrum disorders. Special attention was paid to siblings, given the increased risk of ASDs in first-degree relatives.

(d) The psycho-social and familial factors were taken into consideration within the perspective of evaluating environmental resources and the specific vulnerabilities of the family group. The coordinating role of the consultant was particularly useful in the perspective of a global evaluation, not purely limited to medical or psychological aspects.

(e) Recapitulation of previous actions: the previous evaluations and the child's response to any treatment program had to be reviewed.

\section{Pediatric Examination}

(a) Principle objectives: check for pathologies that would likely respond to medical treatment (epilepsy for example), and/or that had important consequences on the family level (genetic predisposition such as $\mathrm{X}$ fragile or tuberous sclerosis of Bourneville). (b) The medical examination: check for pathologies frequently associated with autism, which could guide the examination and laboratory investigations. Neurological assessment focusing on symptoms that suggest seizures and a careful review of neurological status were indicated. Longitudinal measurements of head circumference, examination for dysmorphic or unusual features, neurocutaneous abnormalities checked with a Wood's lamp examination, tone, gait, and a cranial nerves examination were also indicated.

(c) The audiological and visual examinations were priorities. If an audiologic examination was conducted, other auditory tests would be required if there was any doubt as to audition. In the same manner, a vision examination would be carefully done.

\section{Child Psychiatric Observation}

Should take into account the environmental aspects that would be likely to have an impact on the child's behavior, and that would not necessarily be the same in the consultation, at the day nursery, or at home. The clinician should be attentive to the factors that have a positive or negative influence on the participation of the child. His way of relating to others and his manner of communication should be carefully taken into account. Observations of the child interacting with his parents should furnish indications not only of the child, but also of the parental response to the stress connected to the child's symptoms. Several sessions are generally necessary for observation.

Special attention should be paid to developmental issues. Characteristic symptoms in the areas of social interaction, language and communication, and restricted or unusual interests and behaviors, should be evaluated, as should associated behavioral difficulties, such as self-injury and aggression.

We proposed the use of diagnostic assessment scales, which we normally used.

\section{For Children over 18 Months Old}

A videotaped consultation, with a period of relatively free examination of the child, and another period for the use of a standardized diagnostic tool (with the Childhood Autism Rating Scale, CARS). 


\section{For Children Less than 18 months Old}

A period of free examination, associated with the use of a diagnostic observation tool, such as the ERCA-N (Abbreviated evaluation of the autistic behavior of the newborn) or the PL-ADOS (PreLinguistic Autism Diagnostic Observation Schedule).

\section{Psychological Assessment}

Included the use of various tools

(a) Developmental and intelligence testing: the choice of the test could vary according to the characteristics of the child (age, development level, language...). Brunet-Lézine, Borel-Maisonny, Termann-Merrill...

(b) Adaptive and behavioral assessment: more specific tests (PEP-R: Psycho Educational Profile Revised; ECSP: scale of early social communication by Guidetti and Tourette).

(c) Speech-language assessment: evaluating vocabulary, language skills, oral-motor skills and pragmatic skills.

The choice of tests was motivated by their interest in the evaluation of different abilities, the possibility of isolating relatively homogenous subgroups, and their large-scale use that allowed comparative studies. They are also adapted to the profile of the child. There is no arbitrary systematization that requires each child to go through the same battery of tests.

\section{Other Assessments and Laboratory Investigations}

These were done according to the signs presented by the child. If none of these exams were carried out systematically, we could nonetheless distinguish a certain hierarchy in their importance.

\section{Systematically Done}

- Audiologic assessment

\section{Commonly Done}

- Electroencephalography (checking signs of epilepsy) that includes a marked course of prolonged sleep patterns (systematically assessed)

- genetic data (checking X fragile in particular) for genetic counseling
- cerebral imaging (MRI)

- electrophysiological data (cortical or brain stem evoked potential)

- occupational and physical therapy assessment

- routine laboratory studies with lead level

Other Foreseeable Examinations, such as:

- biochemical assessment checking metabolic abnormalities

- study of the metabolism of the neurotransmitters

\section{Diagnostic Recapitulation}

An important stage where the different elements of the assessment are synthesized, where hypotheses are formulated as well as a diagnosis allowing for proposals of a treatment plan. An important role is devoted to the clinician who is supposed to assume an overall coordination of assessments and liaison with different providers of intervention.

\section{Treatment Plan}

An appropriate treatment plan was proposed at the end of the diagnostic step according to the elements of this assessment. The treatment chosen depended to a large extent on the wishes of the parents, and the effective orientation depended on the available options offered in each therapeutic area. The treatment plan addressed goals for educational intervention, target symptoms, and co-morbid conditions, and monitored various functional areas.

Schematically, the types of treatment available locally were the following, and were not necessarily exclusive:

- Institutional treatment plan in a child psychiatric setting (internal therapeutic day hospital, half-time therapeutic treatment center, partial hospitalization)

- Institutional treatment in a pedagogic-educative or specialized re-educative setting

- School integration in an ordinary setting with a specialized pedagogic aid

- Parent-infant therapeutic consultations

- Individual psychotherapeutic treatment

- Treatment focused on physical issues (physical therapy, sensorial integration) 
- Speech-language therapy

- Drug treatment

No treatment has been proven obviously superior in terms of therapeutic results. Nor are there formal elements that would permit us to exclude one or another form of treatment, arguing that they are ineffective or even harmful. Nor is there a clear consensus in favor of a specific method, but there is evidence to support a multidisciplinary therapeutic approach.

We have not found elements in the medical literature that would allow us to determine a specific therapeutic orientation relative to a pathological profile found in a framework of data. It is therefore not possible now to propose practical recommendations for a global therapeutic orientation. This does not reduce the importance of the initial assessment, which allows us to specify the diagnosis and spot dysfunction in certain areas. This orients the course of therapy within an established treatment framework. Further research is necessary in order to evaluate the various therapeutic possibilities and their respective indications.

The type of treatment usually recommended is multidimensional, taking into account the different aspects of the pathology (educative, social, and emotional). Whatever the etiopathogenic model of these problems might be, it seems important to us to keep a broad perspective that is not limited to an exclusive framework of understanding the problem (for example, autism understood as being a deficit or handicap that would justify only an educative or rehabilitation type of treatment). A broad perspective should integrate other points of view (such as the notion of autistic defenses, or even the factors that maintain the pathology at the core of the family). The absence of scientific validation for the etiopathogenic model on which the treatment is based requires caution and an open mind in selecting treatments, and a certain rigor in their evaluation. Further clinical research should be focused on the evaluation of different types of treatment based on clinical outcomes.

\section{APPENDIX B}

Vaud demographics and child and adolescent psychiatry global activity.

The Canton de Vaud has 628,000 inhabitants and 7500 births a year. A quarter of this population is under 19 years of age. Each year, a total of 5700 children were followed. Overall, there were 55,000 consultations and 3,900 new cases per year in the public sector.

The canton of Vaud includes 103 child and adolescent psychiatrists (about $2 / 3$ of whom work in public clinics while the other $1 / 3$ are in private practice), 97 pediatricians and 539 general practitioners.

\section{REFERENCES}

American Academy of Child and Adolescent Psychiatry Official Action. (1999). Practice parameters for the assessment and treatment of children, adolescent, and adults with autism and other pervasive developmental disorders. Journal of The American Academy of Child \& Adolescent Psychiatry, 38, S32-S54.

Baird, G., Charman, T., Baron-Cohen, S., Cox, A., Swettenham, J., Wheelwright, S., et al. (2000). A screening instrument for autism at 18 months of age: A 6-year follow-up study. Journal of the American Academy of Child Adolescent Psychiatry, 39, 694-702.

Baron-Cohen, S., Allen, J., \& Gillberg, C. (1992). Can autism be detected at 18 month? British Journal of Psychology, 161, 839843.

Baron-Cohen, S., Cox, A., Baird, G., Sweetenham, J., Nightingale, K.M., Drew, A., et al. (1996). Psychological markers in the detection of autisme in infancy in a large population. British Journal of Psychology, 168, 158-163.

Burnand, B. (1999). Des recettes de cuisines utiles? Courrier du Médecin Vaudois, 6, 3-5.

Cabana, M. D., Rand, C. S., Powe, N. R., Wu, A. W., Wilson, M. H., Abboud, P. A., et al. (1999). Why don't physicians follow clinical practice guidelines? A framework for improvement. The Journal of the American Medical Association (JAMA), $282,1458-1465$.

Danion-Grilliat, A. (1996). Autism in children. Diagnosis, course, treatment. Revue du Praticien, 46, 2361-2366.

Davis, D. A., \& Taylor-Vaisey, A. (1997). Translating guidelines into practice. A systematic review of theoretic concepts, practical experience and research evidence in the adoption of clinical practice guidelines. Canadian Medical Association Journal (CMAJ), 157, 408-416.

De Giacomo, A., \& Fombonne, E. (1998). Parental recognition of developmental abnormalities in autism. European Child \& Adolescent Psychiatry, 7, 131-136.

Dilavore, P. C., Lord, C., \& Rutter, M. (1995). The pre-linguistic autism diagnostic observation schedule. Journal of Autism and Developmental Disorders, 25, 355-379.

Filipek, P. A., Accardo, P. J., Ashwal, S., Baranek, G. T., Cook, E. H., Dawson, G., et al. (2000). Practice parameter: Screening and diagnosis of autism - Report of the Quality Standards Subcommittee of the American Academy of Neurology and the Child Neurology Society. Neurology, 55, 468-479.

Filipek, P. A., Accardo, P. J., Baranek, G. T., Cook, E. H. Jr., Dawson, G., Gordon, B., et al. (1999). The screening and diagnosis of autistic spectrum disorders. Journal of Autism and Developmental Disorders, 29, 439-484.

Fombonne, E. (1999). The epidemiology of autism: A review. Psychological Medicine, 29, 769-786.

Fombonne, E., Simmons, H., Ford, T., Meltzer, H., \& Goodman, R. (2001). Prevalence of pervasive developmental disorders in the British nationwide survey of child mental health. Journal of the American Academy of Child Adolescent Psychiatry, 40, $820-827$.

Gillberg, C., \& Wing, L. (1999). Autism: Not an extremely rare disorder. Acta Psychiatrica Scandinavica, 99, 399-406. 
Gilman, J. T., \& Tuchman, R. F. (1995). Autism ans associated behavioral disorders: Pharmacotherapeutic intervention. Annals of Pharmacotherapy, 29, 47-56.

Gray, K. M., \& Tonge, B. J. (2001). Are there early features of autism in infants and preschool children? Journal of Paediatrics and Child Health, 37, 221-226.

Graz, B., Vader, J. P., Burnand, B., \& Paccaud, F. (1996). Quality assurance in Swiss University Hospitals: A survey among clinical department heads. International Journal for Quality in Health Care, 8, 271-277.

Gresham, F. M., \& MacMillan, D. L. (1997). Early Intervention Project: Can its claims be substantiated and its effects replicated? Journal of Autism and Developmental Disorders, 28, 5-13.

Hayward, R. S. (1997). Clinical practice guidelines on trial. Canadian Medical Association Journal (CMAJ), 156, 1725-1727.

Hoagwood, K., \& Olin, S. S. (2002). The NIMH blueprint for change report: Research priorities in child and adolescent mental health. Journal of the American Academy of Child Adolescent Psychiatry, 41, 760-767.

Holzer, L. (2000). «Dépistage des troubles envahissants du développement», Holzer L. Petite Enfance, Juin 2000, 34-41. Petite Enfance, 74, 34-41.

Jellinek, M., \& Little, M. (1998). Supporting child psychiatric services using current managed care approaches: You can’t get there from here. Archives of Pediatrics \& Adolescent Medicine, $152,321-326$

Jellinek, M. S. (1999). Changes in the practice of child and adolescent psychiatry: Are our patients better served? Journal of Developmental and Behavioral Pediatrics, 20, 378-380.

Koegel, R. L., Koegel, L. K., \& McNerney, E. K. (2001). Pivotal areas in intervention for autism. Journal of Clinical Child Psychology, 30, 19-32.

Lord, C. (1997). Diagnostic instruments in autism spectrum disorders. In D. J. Cohen, \& F. R. Volkmar (Eds.), Autism and pervasive developmental disorders. (pp. 460-483). New-York: Wiley.

Mandell, D. S., Listerud, J., Levy, S. E., \& Pinto-Martin, J. A. (2002). Race differences in the age of diagnosis of autistic disorder. Abstract 33251

McEachin, J., Smith, T., \& Lovaas, I. (1993). Long-term outcome for children with autism who received early intensive behavioral treatment. American Journal on Mental Retardation, 97, 359-372.

Misès, R., Fortineau, J., Jeammet, P., Lang, J., Mazet, P., Plantade, A., \& Quemada, N. (1988). Classification française des troubles mentaux de l'enfant et de l'adolescent. Psychiatr Enfant, 31, 67-135.
Ozonoff, S., \& Cathcart, K. (1998). Effectiveness of a home program intervention for young children with autism. Journal of Autism and Developmental Disorders, 28, 25-32.

Reid, W. H., Balis, G. U., \& Sutton, B. J. (1997). Pervasive developmental disorders. In The treatment of psychiatric disorders, third edition revised for DSM IV (pp. 441-447). Bristol: Brunner/Mazel publishers.

Robins, D. L., Fein, D., Barton, M. L., \& Green, J. A. (2001). The modified checklist for autism in Toddlers: An initial study investigating the early detection of autism and pervasive developmental disorders. Journal of Autism and Developmental Disorders, 31, 131-144.

Rodrigues-Dagaeff, C., Junier, L., Dessiex, V., Holzer, L., MullerNix, C., \& Ansermet, F. (1999). Autistic disorders: Screening and early intervention. Revue Medicale de la Suisse Romande, $119,111-115$.

Rutter, M. (1999). The Emanuel Miller Memorial Lecture 1998. Autism: Two-way interplay between research and clinical work. Journal of Child Psychology Psychiatry, 40, 169-188.

Sauvage, D., \& Hameury, L. (1990). Interventions précoces. In P.J- Parquet, C. Bursztejn, \& B. Golse (Eds.), Soigner, éduquer l'enfant autiste. Paris: Masson.

Schopler, E., Reichler, R. J., DeVellis, R. F., \& Daly, K. (1980). Toward objective classification of childhood autism: Childhood Autism Rating Scale (CARS). Journal of Autism and Developmental Disorders, 10, 91-103.

Sigman, M. (1999). Change and continuity in the development of children with autism. Newsletter of the World Association for Infant Mental Health, 7, 1-14.

Steernemann, P., Muris, P., Merckelbach, H., \& Willems, H. (1997). Assessment of development and abnormal behavior in children with PDD. Evidence for the reliability and validity of the revised psychoeducational profile. Journal of Autism and Developmental Disorders, 27, 177-185.

Stella, J., Mundy, P., \& Tuchman, R. (1999). Social and nonsocial factors in the Childhood Autism Rating Scale. Journal of Autism and Developmental Disorders, 29, 307-317.

Stone, W. L., Lee, E. B., Ashford, L., Brissie, J., Hepburn, S. L., Coonrod, E. E., et al. (1999). Can autism be diagnosed accurately in children under 3 years? Journal of Child Psychology and Psychiatry, 40, 219-226.

Tanguay, P. E., Robertson, J., \& Derrick, A. A. (1998). A dimensional classification of autism spectrum disorder by social communication domains. Journal of the American Academy of Child and Adolescent Psychiatry, 37, 271-277. 